\title{
Echinometra lucunter (Linnaeus) (Echinoidea, Echinometridae) als Wirt einer komplexen Lebensgemeinschaft im Karibischen Meer
}

\author{
Sabine Schoppe \\ Institut für Allgemeine und Spezielle Zoologie, Justus-Liebig-Universität; Stephanstr. 24, \\ D-W-6300 Gießen, Bundesrepublik Deutschland
}

\begin{abstract}
Echinometra lucunter (Linnaeus) (Echinoidea, Echinometridae) as host of a complex association in the Caribbean Sea. The association between Acyrtus rubiginosus (Gobiesocidae), Clastotoechus vanderhorsti (Porcellanidae), Ophiothrix sp. (Ophiotrichidae) and their rock-boring host Echinometra lucunter (Echinometridae) has been studied on the rocky shores of Santa Marta, Colombia. The three inhabitants of the sea urchin dwellings benefit from their cohabitation by receiving effective shelter from intertidal effects and from predators. The sea urchin, by contrast, seems neither to be benefitted nor harmed by the association.
\end{abstract}

\section{EINLEITUNG}

Das Felslitoral gehört zu den artenreichsten Großbiotopen des marinen Benthos. Die Hauptursache dafür ist in der Stabilität der Substrate zu suchen, die sessilen und halbsessilen Organismen einen geeigneten Lebensraum in Form von Verankerungsmöglichkeiten bieten. Durch die starke Besiedelung erfährt die Oberfläche des Gesteins eine zusätzliche Gliederung, wodurch wieder neue ökologische Nischen entstehen. Das Nebeneinander von vielen Arten auf engstem Raum führt zu einer ständigen Konkurrenz um Lebensraum, unter Umständen aber auch zu einem toleranten Zusammenleben der dort ansässigen Arten.

Echinometra lucunter gehört, wie auch einige andere Vertreter der Familie Echinometridae, zu den felsbohrenden Seeigeln. Seine Lebensweise in exponierten Bereichen des Felslitorals ist durch auffällige Anpassungen an diesen Lebensraum geprägt. Aus seiner Lebensweise resultiert eine sekundäre Strukturierung der Felsoberfläche und damit neuer Lebensraum für weitere Litoralarten. Die besonderen Lebensbedingungen im Litoral (Brandungseffekte, Predatorendruck) und die begrenzte Verfügbarkeit von Platz führen im untersuchten Fall zu einer Assoziation zwischen vier der ansässigen Spezies.

Schon Lewis (1960) und Markham (1975) geben Hinweise auf eine Vergesellschaftung von E. lucunter mit dem Porzellaniden Clastotoechus vanderhorsti (Schmitt). Werding (1983) konnte dies für das Gebiet um Santa Marta bestätigen. Ein Hinweis für das Zusammenleben des Seeigels mit dem Gobiesociden Acyrtus rubiginosus (Poey) in St. Croix (Jungfern-Inseln) gibt Teytaud (1971). Acero \& Garzon (1987) fanden den Fisch 
dann auch mehrmals im Felslitoral von Santa Marta in den Bohrlöchern von E. lucunter. Manjarres (1984) vermerkt ferner das Auftreten eines Schlangensternes, Ophiothrix sp., in den Bohrlöchern von E. lucunter.

Als Karpose wird im folgenden eine interspezifische Vergesellschaftung bezeichnet, bei der die eine Art Nutzen, die andere Art, der Wirt, hingegen keinen Schaden erleidet (Matthes, 1967). Man spricht von Synökie, wenn der Nutzen des Karponten in einem eindeutigen Überlebensvorteil durch das Mitbenutzen der schutzgebenden Wohnung besteht. Kommensalismus ist dann gegeben, wenn ein zusätzlicher Profit aus Nahrungsüberschuß und Abfallstoffen des Wirtes erzielt wird.

\section{MATERIAL UND METHODEN}

Während einer sechsmonatigen Untersuchung (November 1988 bis Mai 1989) der Seeigelpopulationen von Echinometra lucunter im Felslitoral von Sanța Marta, Kolumbien, wurde besonderes Augenmerk auf die Vergesellschaftung des Seeigels mit anderen Litoralarten gelegt.

Versuche und Beobachtungen sollten einerseits die Art der Beziehung zwischen $E$. lucunter und den Assoziationspartnern und andererseits das Verhältnis der Mitbewohner untereinander klären.

Bei der Bewertung von Lebensgemeinschaften ergibt sich oft das Problem der qualitativen Zuordnung zu einem definierten Vergesellschaftungstyp. Die Schwierigkeiten sind einerseits in der Komplexität vieler Lebensgemeinschaften begründet, andererseits in der Schwierigkeit der direkten bzw. längeren Beobachtung insbesondere im Felslitoral.

Die Ergebnisse basieren auf Freilanduntersuchungen, quantitativem Sammeln und Beobachtungen an gehälterten Tieren.

Ausgangspunkt für alle Exkursionen war das Instituto de Investigaciones Marinas de Punta de Betín (INVEMAR) am äußeren Ende der Halbinsel Punta de Betín, wo auch die Laborarbeiten stattfanden. In Abbildung 1 sind die Sammelstellen Punta de Betín, Taganga, Granate und Nenguange gekennzeichnet. Sowohl die Materialsammlungen als auch die Feldbeobachtungen wurden tauchend mit Schnorchel durchgeführt.

\section{ERGEBNISSE}

Echinometra lucunter besiedelt Felsspalten oder bohrt Löcher in den felsigen Untergrund des Eulitorals und oberen Sublitorals. Er bildet dort ausgedehnte Populationen. Die Bohrlöcher schützen ihn vor den starken Brandungswellen. Im exponierten Bereich des Felslitorals konnte nie beobachtet werden, daß E. lucunter seine Bohrlöcher verläßt. Seine Lebensweise hat somit einen direkten Einfluß auf seine Ernährung. Er ernährt sich von mit der Wasserbewegung angetriebenen Algen, die er mit Hilfe der Stacheln einfängt. Die Algenfragmente werden durch Überkreuzen der Stacheln festgehalten und dann mit Hilfe der Stacheln und Ambularkralfüßchen zum Kauapparat befördert.

Die sedentäre Lebensweise von $E$. lucunter wird im Untersuchungsgebiet regelmäBig von dem Knochenfisch Acyrtus rubiginosus, dem anomuren Krebs Clastotoechus vanderhorsti und dem Schlangenstern Ophiothrix sp. genutzt. Sie suchen in den von $E$. lucunter bewohnten Bohrlöchern Schutz vor der Brandung und vor Freßfeinden. Sie alle 


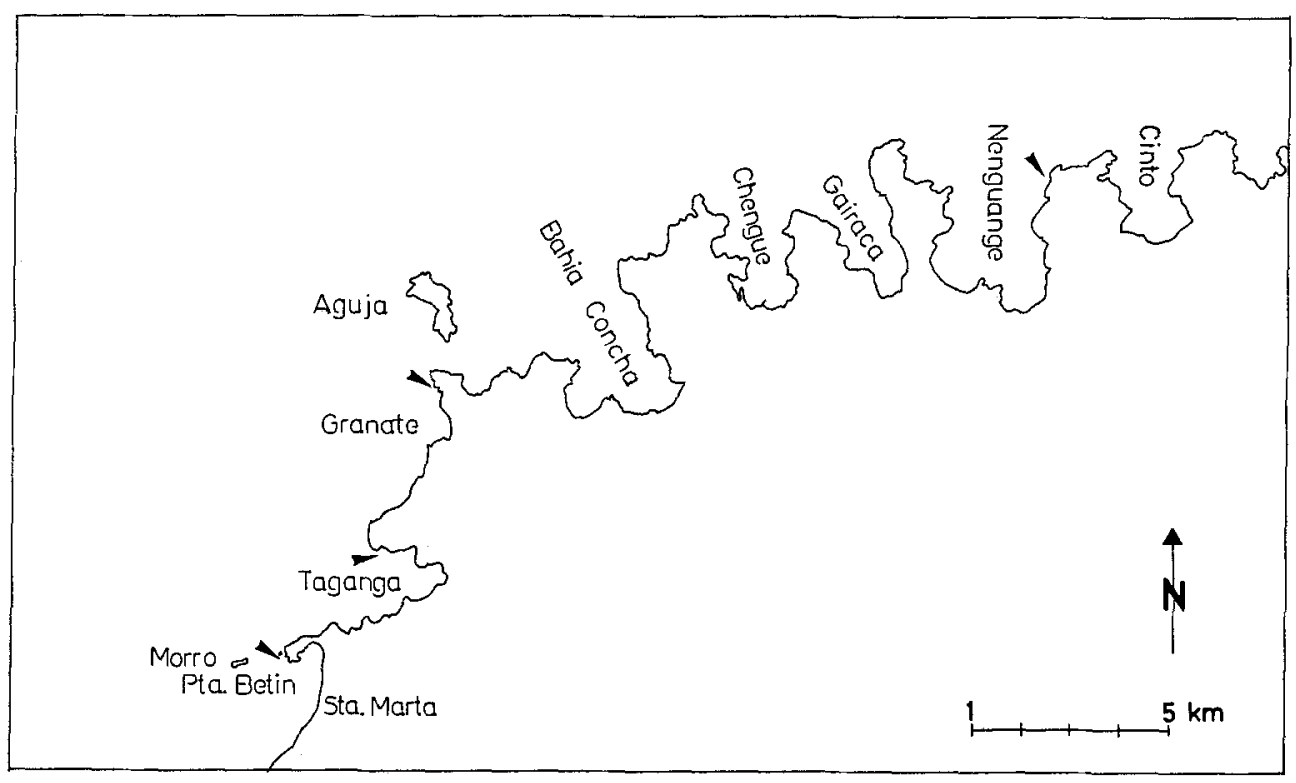

Abb. 1. Das Untersuchungsgebiet im Karibischen Meer: Santa Marta und der angrenzende Parque Nacional de Tairona

sitzen in den Bohrlöchern unter dem Seeigel oder an den seitlichen Wänden des Bohrloches, dem Substrat zugewandt (Abb. 2).

In keinem Fall wurden die Tiere auf dem Seeigel, d.h. zwischen seinen Stacheln gefunden. Alle drei genannten Mitbewohner wurden im Untersuchungsgebiet nie ohne ihren Wirt angetroffen. Die Häufigkeit und Regelmäßigkeit, mit der sie bei $E$. lucunter beobachtet werden konnten, war sehr hoch (Tabelle 1).

Tabelle 1. Mittelwerte der Ergebnisse aller vier Sammelstellen (Punta de Betín, Taganga, Granate und Nenguange)
E. lucunter allein
A. rubiginosus
C. vanderhorsti
Ophiothrix sp.
Alle drei Mitbewohner
in 22 von 258 Bohrlöchern $=8,5 \%$
in 108 von 258 Bohrlöchern $=41,9 \%$
in 160 von 258 Bohrlöchern $=62,0 \%$
in 166 von 258 Bohrlöchern $=64,3 \%$
in 44 von 258 Bohrlöchern $=17,1 \%$

Dies unterscheidet sie eindeutig von anderen Litoralarten, die nur sporadisch in den Bohrlöchern des Seeigels gefunden wurden und die keine Affinität zu dem Seeigel zeigen.

Die drei Mitbewohner zeigen eine hohe interspezifische Toleranz. Wie die Abbildung 3 zeigt, wurde die Kombination E. lucunter mit C. vanderhorsti und Ophiothrix sp. am häufigsten $(23,2 \%)$ angetroffen. Dies begründet sich in dem etwas selteneren Vorkommen von $A$. rubiginosus. Die im Freiland beobachtete hohe interspezifische 


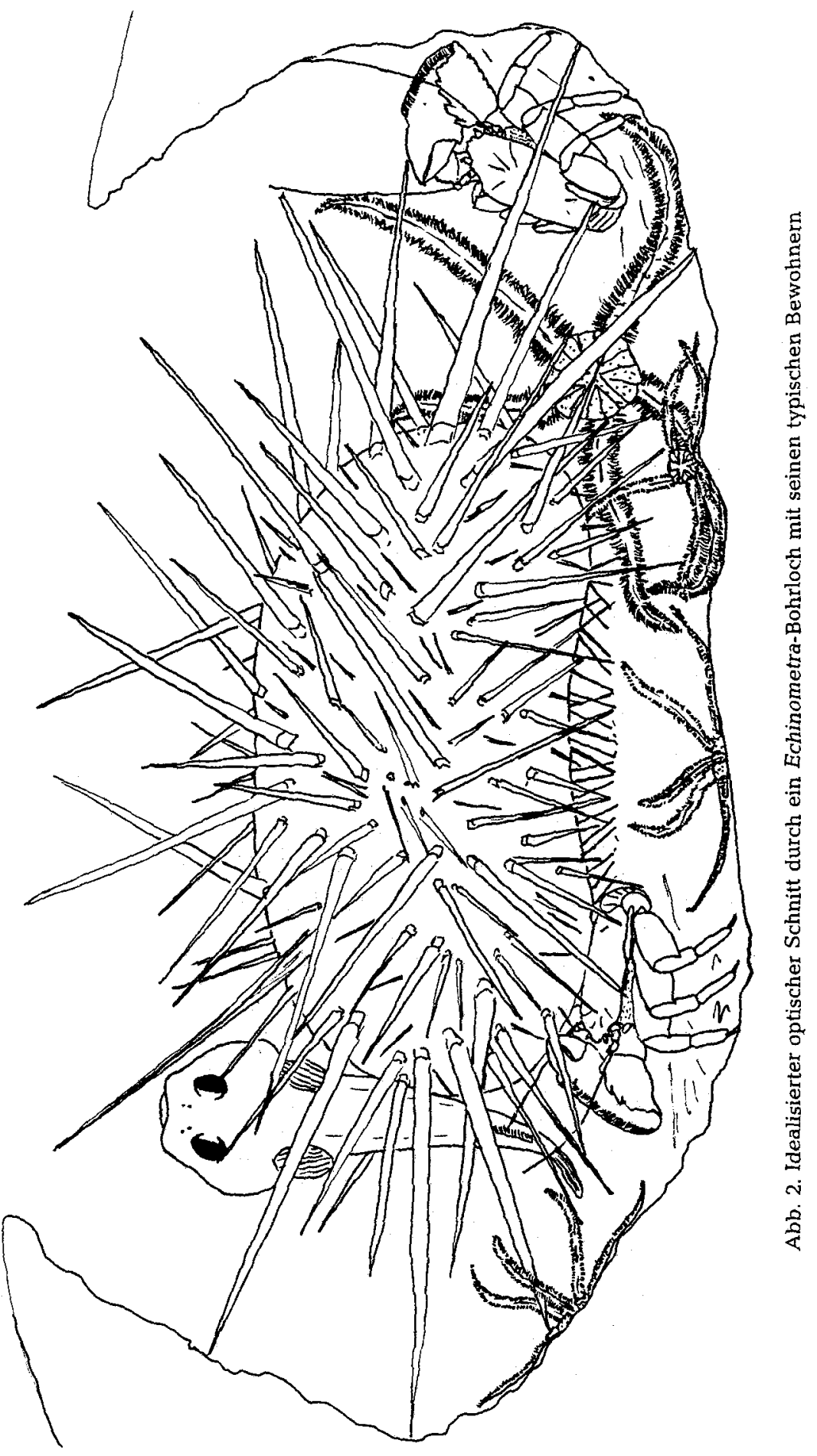




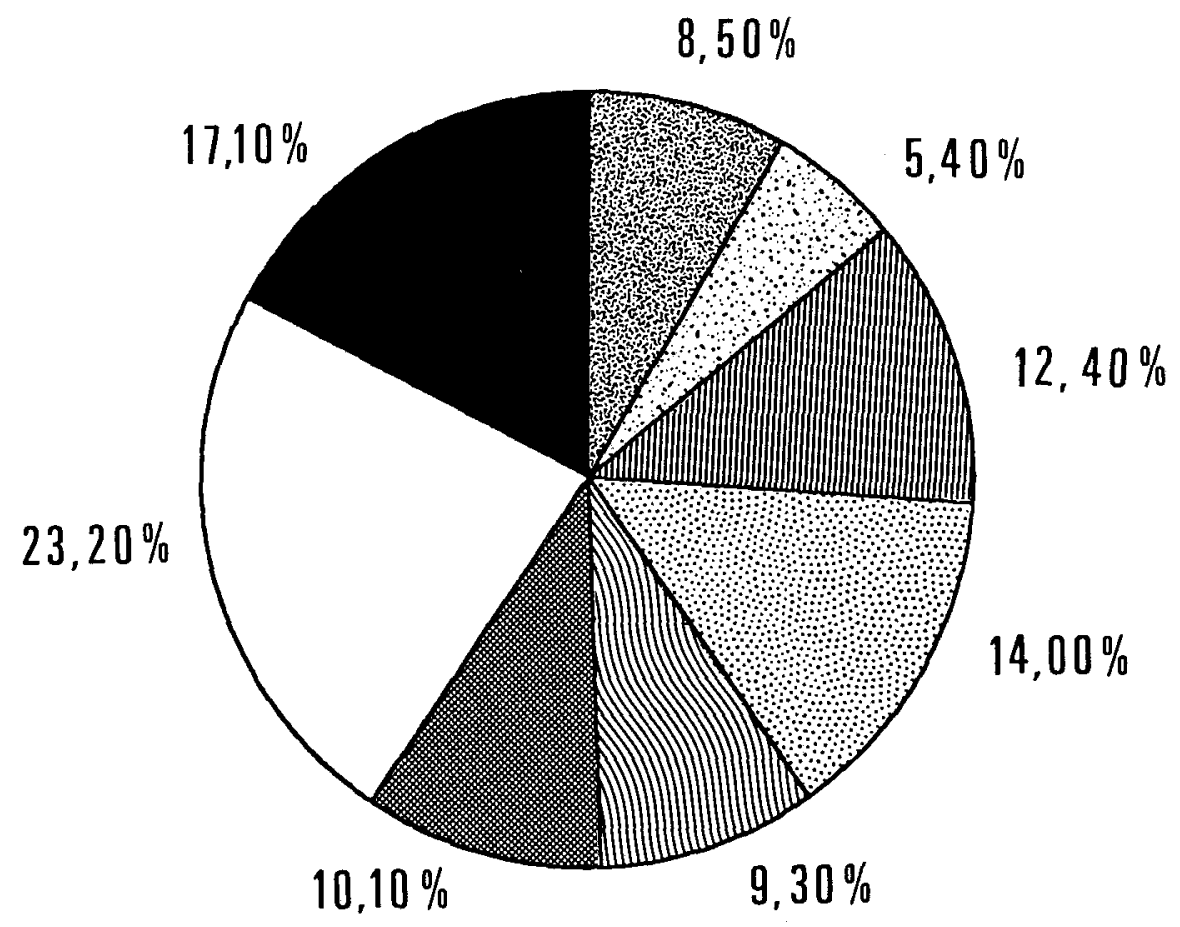

E. lucunter allein

E. lucunter \& A. rubiginosus

西同 E. lucunter \& C. vanderhorsti

E. lucunter \& Ophiothrix sp.

iㅣㄴ E. lucunter \& A. nubiginosus \& C. vanderhorsti

E. lucunter \& A. rubiginosus \& Ophiothrix sp.

E. lucunter \& C. vanderhorsti \& Ophiothrix sp.

E. lucunter \& A. rubiginosus \& C. vanderhorsti \& Ophiothrix sp.

Abb. 3. Graphische Darstellung aller unter den Assoziationspartnern angetroffenen Kombinationsmöglichkeiten. Die Prozentwerte stellen die gemittelten Häufigkeitswerte der Bewohner für die Untersuchungsorte Punta de Betín, Taganga, Granate und Nenguange dar 
Toleranz konnte durch Aquarienversuche bestätigt werden. Zusätzlich durchgeführte Nahrungsanalysen zeigten, daß aufgrund der unterschiedlichen Ernährungsweise und Nahrung eine Nahrungskonkurrenz ausgeschlossen werden kann.

Die nachfolgenden Ergebnisse der Freilandbeobachtungen konnten ebenfalls durch Experimente in Aquarien bestätigt werden. C. vanderhorsti besiedelt die Bohrlöcher meist paarweise $(61,9 \%)$ und duldet neben seinem Geschlechtspartner nur juvenile Krebse der gleichen Art. A. rubiginosus bewohnt die Bohrlöcher allein, der Geschlechtspartner wird vermutlich nur während der Paarungszeit aufgesucht. Ophiothrix sp. zeigt kein aggressives Verhalten gegenüber Individuen der gleichen Art.

Die spezifische Affinität von $A$. rubiginosus und $C$. vanderhorsti zu E. lucunter ist so groß, daß sie auch im Aquarium immer dessen Gesellschaft aufsuchen. Ophiothrix sp. versteckt sich im Aquarium unter Steinen. Aquarienversuche mit E. lucunter, E. viridis, Seeigeln anderer Gattungen und Attrappen haben gezeigt, daß A. rubiginosus den Seeigel anhand optischer Merkmale erkennt, wobei kurze dunkle Stacheln ausschlaggebend zu sein scheinen. Auch $C$. vanderhorsti erkennt den Seeigel anhand optischer Merkmale, die durch taktile Erkennungsmechanismen unterstützt werden. Ophiothrix sp. hingegen ist auf rein taktile Erkennungsmechanismen angewiesen. Verliert der Schlangenstern den Kontakt zu seinem Seeigel (z. B. in Aquarienversuchen), so ist es für ihn sehr viel schwieriger bzw. zufallsbedingt, wieder einen Seeigel zu finden.

Bei der untersuchten Karpose handelt es sich im speziellen um eine Synökie. Die Synöken erlangen durch die Mitbenutzung der Wohnung ihres Wirtes einen effektiven Schutz vor Freßfeinden und der starken Wasserbewegung. Ophiothrix sp. steht zusätzlich in einem kommensalischen Verhältnis zu seinem Wirt und den anderen Mitbewohnern, da er sich außer von Detritus vom Nahrungsüberschuß seines Wirtes und den Abfallprodukten aller drei weiteren Bewohner ernährt. Im Untersuchungsgebiet muß das Synökie-Verhältnis für alle drei Karponten als obligatorisch angesehen werden.

\section{DISKUSSION}

Echinometra lucunter hat sich durch die Fähigkeit, Löcher in den Fels zu bohren, ein Biotop erschlossen, in dem das Leben sonst für ihn unmöglich wäre. Seine sedentäre Lebensweise in Bohrlöchern hat $z u$ erstaunlichen Anpassungen in bezug auf seine Ernährung geführt. Die Lebensweise und die Art des Nahrungserwerbes stehen in unmittelbarem Zusammenhang.

Seine charakteristische Lebensweise eröffnet ferner anderen Litoralarten neuen Lebensraum. Die Karponten von E. lucunter haben sich diese Gegebenheit zunutze gemacht und teilen seine "Wohnung" mit ihm. Nur wenn der Seeigel, wie im untersuchten Fall, sein Bohrloch nicht verläßt, wird den Mitbewohnern der Schutz gewährleistet, den sie in dieser Wohn- und Schutzgemeinschaft suchen. Besonders deutlich wird dies, wenn $E$. lucunter aus seinem Bohrloch entfernt wird. Wenn es den Mitbewohnern nicht rechtzeitig gelingt, unter einem benachbarten $E$. lucunter Zuflucht zu finden, werden sie sofort von einem der zahlreichen Fische des Gezeitenbereiches gefressen.

Auch wenn angenommen werden kann, daß alle drei Karponten unter bestimmten Lebensbedingungen (verminderter Feinddruck, schwache Wasserbewegung) ohne ihren Wirt überleben können, so scheint doch das Vergesellschaftungsschema bereits evolutiv verankert zu sein. 
Die mit $E$. lucunter vergesellschafteten Arten zeigen zwar keine sichtbaren morphologischen Anpassungen an ihren Wirt, dennoch benötigen die Assoziationspartner spezifische Anpassungen an das Leben ihres.Wirtes, an den Wirt selbst und solche, die ein Auffinden des Wirtes überhaupt ermöglichen. Jeder der Synöken hat Verhaltensweisen entwickelt, die dem Leben in einer speziellen ökologischen Nische angepaßt sind und zu einer besonderen Besiedlungsstrategie führen.

Bei der vergleichenden Betrachtung der spezifischen Affinität der drei Mitbewohner zu ihrem Wirt läßt sich feststellen, daß C. vanderhorsti, A. rubiginosus und Ophiothrix sp. unter natürlichen Bedingungen eine annähernd gleich große Affinität zeigen. Ihre hohe Spezifität bezieht sich nicht auf die Wirtsart allein, sondern auch auf die charakteristische Lebensweise ihres Wirtes in Bohrlöchern des Eulitorals und oberen Sublitorals. Bedingt durch den (je nach Art unterschiedlichen) Mechanismus der Wirtsfindung (optisch, optisch und taktil sowie rein taktil), kann allerdings Ophiothrix sp. in der Aquarienhälterung einem mobilen $E$. lucunter nicht mehr folgen.

Danksagung. Dem Instituto de Investigaciones Marinas de Punta de Betín (INVEMAR) und seiner Trägerorganisation COLCIENCIAS (Bogotá) danke ich für die Bereitstellung eines Arbeitsplatzes und die Hilfestellung bei den Ausfahrten, dem Deutschen Akademischen Austauschdienst DAAD für die Teilfinanzierung meiner Reise.

\section{LITERATUR}

Acero, A. \& Garzon, F. J., 1987. Los peces marinos hallados durante la expedición Uraba II al Caribe Chocoano (Colombia). - An. Inst. Invest. mar. Punta de Betín 17, 113-136.

Lewis, J. B., 1960. The fauna of the rocky shores of Barbados, West Indies. - Can. J. Zool. 38, $391-435$

Manjarres, G. A., 1984. Estructura de la macrofauna béntica del litoral rocoso en la region de Santa Marta. Tesis de grado, Universidad Nacional de Colombia, Bogota, $128 \mathrm{pp}$.

Markham, J., 1975. Bopyrid isopods infesting porcellanid crabs in the northwestern Atlantic. Crustaceana 28, 257-270.

Matthes, D., 1967. Die Terminologie interspezifischer (heterotypischer) Beziehungen. - Zool. Anz. $179,313-319$.

Teytaud, A. R., 1971. Food habits of the goby, Ginsburgellus novemlineatus and the clingfish, Arcos rubiginosus, associated with echinoids in the Virgin Island, - Caribb. J. Sci. 11, 41-45.

Werding, B., 1983. Kommensalische Porzellaniden aus der Karibik (Crustacea: Anomura). - Crustaceana $45,1-14$. 